entry into the inflamed synovium and is regarded as a switch from acute to chronic inflammation. The non-canonical nuclear factor kappaB (NF-kB) pathway, with its main regulator NF-kB inducing kinase (NIK), may play a central role in this process.

Objectives To determine the effect of non-canonical NF-kB signalling on pro-angiogenic gene expression in RASF and on the angiogenic potential of human umbilical vein endothelial cells (HUVEC). Methods RASF were stimulated with lymphotoxin $\alpha_{1} \beta_{2}$ (LT) or LIGHT to activate non-canonical NF-kB signalling, and/or TNF to selectively activate the canonical NF-kB pathway. To effectively block the non-canonical pathway, a dominant negative NIK expressing adenovirus (Ad.NIKdn) was used. Changes in pro-angiogenic gene expression were measured by RT-PCR. Furthermore, to determine the effect on EC proliferation, RASF and HUVEC were cocultured in the absence or presence of LT, LIGHT, TNF or VEGF EC were visualised through immunohistochemical staining of CD31, which was then semi-quantitatively scored.

Results Gene expression analysis of RASF revealed an increase in mRNA levels of VCAM- 1 and IL- 6 after stimulation with LT, LIGHT and TNF. Increased expression of IL-8 and MMP-3 was also observed in cells treated with both TNF and LT or LIGHT. These levels were attenuated in cells transduced with Ad.NIKdn prior to stimulation, indicating that the increased expression levels were at least in part non-canonical NF-kB dependent. CCL2 and bFGF were expressed continuously by RASF regardless of stimulation. In the co-culture, proliferation levels of EC increased under all stimulation conditions, with LIGHT inducing almost a 2-fold increase $(p<0.05)$, which was comparable to VEGF $(p<0.05)$.

Conclusions RASF contribute to synovial angiogenesis through the expression of adhesion molecules, cytokines, chemokines, matrix remodelling enzymes and growth factors. We demonstrate that the non-canonical NF-kB pathway plays an important role in this process by regulating pro-angiogenic genes and promoting EC proliferation. Further investigation of this pathway could lead to novel non-canonical NF-kB blocking therapeutics that inhibit angiogenesis in RA, thereby halting disease progression.

\section{A9.13 TNF-INDUCED- PROTEIN TYROSINE PHOSPHATASE NONRECEPTOR TYPE 2 (PTPN2) AS A NEGATIVE REGULATOR OF INFLAMMATION IN RHEUMATOID ARTHRITIS}

doi:10.1136/annrheumdis-2013-203223.13

'Borbala Aradi, 'Maria Filkova, ${ }^{2}$ Stephanie Kasper, 'Kerstin Klein, ${ }^{2}$ Michael Scharl, 'Beat A Michel, 'Renate E Gay, ${ }^{3}$ Edit I Buzas, 'Steffen Gay, 'Astrid Jüngel. 'Center of Experimental Rheumatology, University Hospital Zürich and Zürich Center for Integrative Human Physiology, Zürich, Switzerland; 'Division of Gastroenterology and Hepatology, University Hospital Zürich and Zürich Center for Integrative Human Physiology, Zürich, Switzerland; ${ }^{3}$ Department of Genetics, Cell- and Immunobiology, Semmelweis University, Budapest, Hungary

Background and Objectives Protein Tyrosine Phosphatase Nonreceptor Type 2 (PTPN2) is a protein tyrosine phosphatase that plays a role in the development of autoimmune diseases. PTPN2 function has not been studied in rheumatoid arthritis (RA), although single nucleotide polymorphisms within the gene have been described to be associated with RA in genome wide association studies. Considering the involvement of PTPN2 in the regulation of key inflammatory pathways, our aim was to analyse the expression and function of PTPN2 in RA synovial fibroblasts (RASF).

Materials and Methods The expression of PTPN2 was assessed in synovial tissue and fibroblasts (passage 4-10) from patients with $\mathrm{RA}$ and osteoarthritis (OA) using immunohistochemistry, real-time PCR (w/o tumour necrosis factor $\alpha$ (TNF $\alpha$ ), IL1 $\beta$, LPS and hypoxia) and Western blotting. PTPN2 was silenced with silencing RNA. Levels of IL-6 and IL-8 expression were measured by commercially available ELISA in cell culture supernatants after silencing PTPN2 in RASF w/o stimulation with tumour necrosis factor $\alpha$ (TNF $\alpha$ ). Apoptosis of RASF was evaluated by AnnexinV staining using flow cytometry after stimulation with TNF-related apoptosis-inducing ligand (TRAIL, $20 \mathrm{ng} / \mathrm{ml}$ ) for 24 hours.

Results In RA synovial tissue, compared with OA, we observed a stronger staining of PTPN2 in both the lining and the sublining layer by immunohistochemistry. On mRNA level we confirmed this overexpression in RA synovial tissue (2.0 fold, $n=4-5)$. In isolated RASF the constitutive mRNA level of PTPN2 was higher than in OASF (1.6 fold, $\mathrm{p}<0.01, \mathrm{n}=10-16$ ).

Levels of PTPN2 were further upregulated in RASF after stimulation with inflammatory cytokines such as TNF $\alpha(10 \mathrm{ng} / \mathrm{ml}$, 24 hours, 3.1 fold, $\mathrm{p}<0.05, \mathrm{n}=4)$, TNF $\alpha$ and IL-1 $\beta(1 \mathrm{ng} / \mathrm{ml}$, 2.3 fold, $\mathrm{n}=5)$, LPS $(100 \mu \mathrm{g} / \mathrm{ml}, 24$ hours, 1.9 fold, $\mathrm{n}=5)$ and by $1 \%$ hypoxia (1.3 fold, $n=3$ ). Accordingly, basal PTPN2 protein expression was 2.0 fold higher in RASF than in OASF $(n=4)$ and TNF $\alpha$ upregulated levels of PTPN2 (1.7 fold). PTPN2-deficient RASF produced 2.4 times more IL- 6 than scrambled siRNA transfected cells (mean \pm SD pg/ml $11412 \pm 6313$ versus $28133 \pm 12734, \mathrm{n}=3$ ). On the other hand, levels of IL-8 were not affected $(35800 \mathrm{pg} / \mathrm{ml}$ versus 24330 $\mathrm{pg} / \mathrm{ml}, \mathrm{n}=3$ ). Furthermore, after silencing, $34 \%$ increase in TRAILinduced apoptosis was detected in RASF $(n=5)$ compared to scrambled controls.

Conclusions Our findings indicate that PTPN2, known to be involved in the pathogenesis of several autoimmune diseases, could be an important negative regulator of inflammation in RASF.

Acknowledgement This work was supported by IMI BTCure, IAR and Masterswitch-FP7.

\section{A9.14 REGULATORY T CELL ABNORMALITIES IN PATIENTS WITH SLE SUGGEST AN IL-2-BASED IMMUNOTHERAPY}

doi:10.1136/annrheumdis-2013-203223.14

${ }^{1,2} \mathrm{C}$ von Spee, ${ }^{2} \mathrm{~N}$ Wassermann, ${ }^{1,2, *} \mathrm{JY}$ Humrich, ${ }^{1,2, *} \mathrm{G}$ Riemekasten. ${ }^{1} \mathrm{German}$ Rheumatism Research Centre, Berlin, Germany; ${ }^{2}$ Charité University Hospital, Rheumatology and Clinical Immunology, Berlin, Germany.

*These authors contributed equally

Background Phenotypic and quantitative abnormalities of regulatory $\mathrm{T}$ cells have been described in association with systemic lupus erythematosus (SLE). Further, an impaired production of IL-2, the essential cytokine for Treg homeostasis, has been described for $\mathrm{T}$ cells from SLE patients. Here, we aim to substantiate the link between IL-2 deficiency and Treg abnormalities in SLE and to provide the basis for an IL-2 based immunotherapy.

Methods Phenotype, frequency and homeostatic status of Foxp3+CD127lo Treg and conventional Foxp3- T cell (Tcon) subsets were analysed by multi-colour flow-cytometry of PBMCs from SLE patients and healthy donors ex vivo and after in vitro low-dose IL-2 treatment. Disease activity was determined according to the SLE activity index (SLEDAI). Two-tailed Mann-Whitney U test or 2-way ANOVA test were used for statistical analysis between patient or treatment groups, Spearman's rank coefficient was used to calculate correlations with disease activity.

Results The frequency of CD25+ cells among Treg was significantly reduced in SLE patients compared to HC. Analysis of Ki67 expression revealed that proliferation was significantly increased in Tcon from SLE patients, resulting in a reduced Treg to Tcon proliferation ratio in SLE patients. The proliferation ratio correlated positively with the frequency of CD25+ Treg and inversely with disease activity. Treatment of SLE PBMCs with low-dose IL-2 in vitro resulted in increased frequencies of CD25+ cells among Treg and increased CD25 expression levels on Treg. Treg, but not Tcon proliferation was significantly increased under low-dose IL-2 treatment compared to untreated controls. 